\title{
Phonon Lateral Confinement Enables Thermal Rectification in Asymmetric Single-Material Nanostructures
}

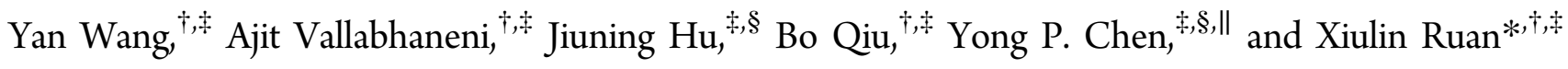 \\ ${ }^{\dagger}$ School of Mechanical Engineering, Purdue University, West Lafayette, Indiana 47907, United States \\ ${ }^{\ddagger}$ Birck Nanotechnology Center, Purdue University, West Lafayette, Indiana 47907, United States \\ ${ }^{\S}$ School of Electrical and Computer Engineering, Purdue University, West Lafayette, Indiana 47907, United States \\ "Department of Physics, Purdue University, West Lafayette, Indiana 47907, United States
}

\section{Supporting Information}

\begin{abstract}
We show that thermal rectification (TR) in asymmetric graphene nanoribbons (GNRs) is originated from phonon confinement in the lateral dimension, which is a fundamentally new mechanism different from that in macroscopic heterojunctions. Our molecular dynamics simulations reveal that, though TR is significant in nanosized asymmetric GNRs, it diminishes at larger width. By solving the heat diffusion equation, we prove that TR is indeed absent in both the total heat transfer rate and local heat flux for bulk-size asymmetric single materials, regardless of the device geometry or the anisotropy of the thermal conductivity. For a deeper understanding of why lateral confinement is

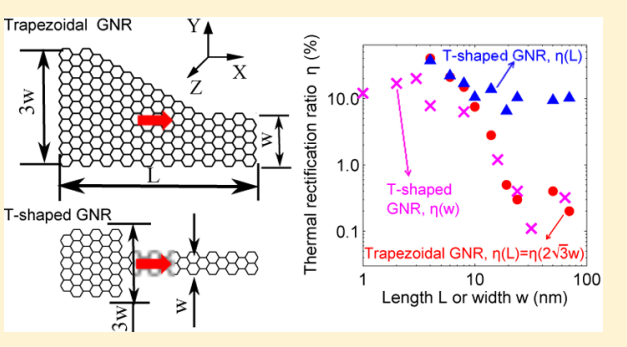
needed, we have performed phonon spectra analysis and shown that phonon lateral confinement can enable three possible mechanisms for TR: phonon spectra overlap, inseparable dependence of thermal conductivity on temperature and space, and phonon edge localization, which are essentially related to each other in a complicated manner. Under such guidance, we demonstrate that other asymmetric nanostructures, such as asymmetric nanowires, thin films, and quantum dots, of a single material are potentially high-performance thermal rectifiers.
\end{abstract}

KEYWORDS: Thermal rectification, phonon lateral confinement, phonon localization, edge/surface effect, molecular dynamics, phonon spectra

I nspired by the impact of electric diodes on the electronics industry, extensive attention has been given to the search of rectification of various other transport processes. ${ }^{1-3}$ Thermal rectification (TR) is a diode-like behavior where the heat current changes in magnitude when the applied temperature $(T)$ bias is reversed in direction. A perfect thermal rectifier would be one that is highly thermal conductive in one direction while insulating in the other, and it is expected to work as a promising thermal management component of electronics as chip size continues decreasing or as a stand-alone thermally driven computing system replacing the electronic ones in certain conditions.

Numerous studies have predicted or demonstrated the existence of TR in bulk or nanosized systems, most of which are heterojunctions (HJ) or graded systems. ${ }^{3-11}$ For twosegment systems, TR was usually attributed to the different $T$ dependence of the thermal conductivity $(\kappa),{ }^{5,7}$ and for interfaces TR has been interpreted as the different phonon spectra mismatch before and after reversing the applied $T$ bias. ${ }^{4}$ Phonon localization was suggested to play a role as well. ${ }^{12,13}$ Recently, TR was also predicted to occur in asymmetric pristine carbon nanostructures, ${ }^{13-17}$ which are composed of a single material and are attractive for their simple structure and high thermal conductance. ${ }^{18}$ However, the origin of TR in such homogeneous nanostructures remains unclear. In this work, we have observed, using molecular dynamics and analytical derivations, that phonon confinement in the lateral dimension is required for TR to occur in asymmetric homogeneous structures made of a single material. We further show that phonon lateral confinement can enable several possible mechanisms which lead to TR, and when the system lateral size becomes large, all of these mechanisms do not hold any more; hence TR disappears. This work reveals the key role played by phonon lateral confinement due to edge/surface effects of phonons in TR in asymmetric homogeneous structures and will provide rational guidance for both theoreticians and experimentalists for the design and application of this new type of thermal rectifier.

First, nonequilibrium molecular dynamics (NEMD) simulations are performed for graphene nanoribbons (GNRs) using the LAMMPS package. ${ }^{19}$ The optimized Tersoff potential ${ }^{20}$ is adopted for modeling $\mathrm{C}-\mathrm{C}$ interactions. Schematics of the supercells are shown in Figure 1a, where the atoms at the two ends are fixed to make a suspended structure, and the free boundary condition is applied to the $Y$ and $Z$ directions. The GNRs are first relaxed in the NVT ensemble for $5 \times 10^{6}$ time

Received: October 10, 2013

Revised: January 3, 2014

Published: January 6, 2014 
(a)

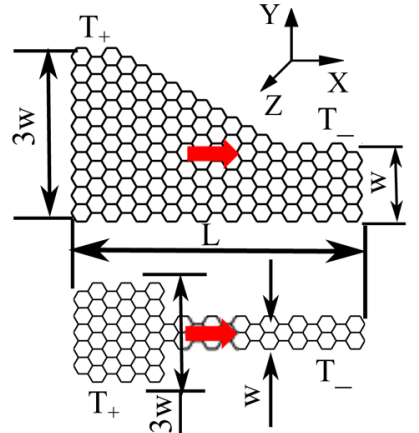

(c)

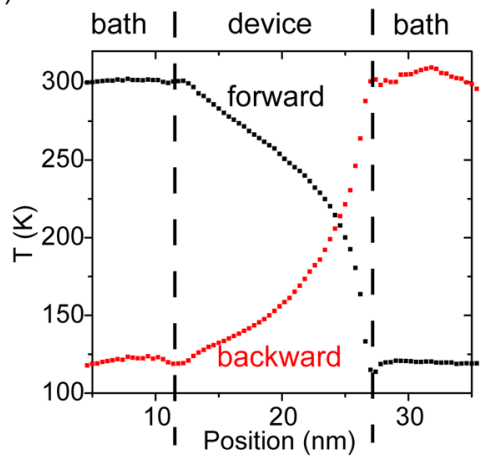

(b)

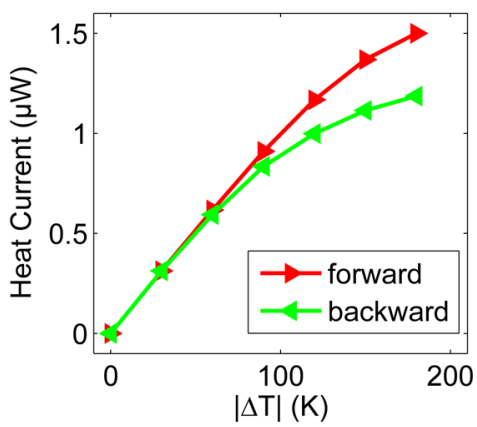

(d)

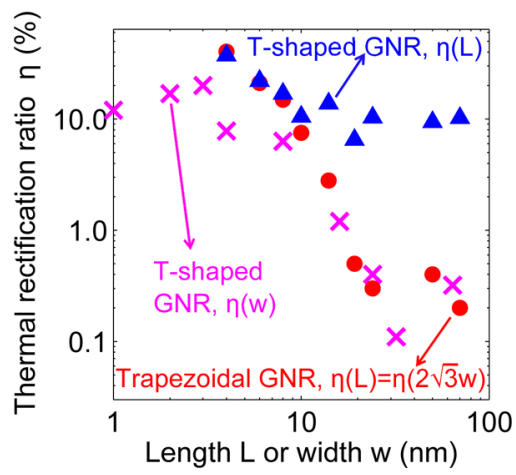

Figure 1. (a) Geometrical definition of the length $(L)$ and width $(w)$ of the trapezoidal-GNRs and T-shaped GNRs; the width ratio of the GNRs is maintained at $3: 1$, and the forward direction is indicated by the arrows. (b) Heat current in a trapezoidal-GNR as a function of $|\Delta T|$. (c) Temperature profiles in the trapezoidal-GNRs. (d) $\eta$ as a function of the length or width of GNRs. $w=2 \mathrm{~nm}$ when $L$ varies, $L=6 \mathrm{~nm}$ when $w$ varies for the T-shaped GNRs, and $L=2 \sqrt{ } 3 w$ for the trapezoidal-GNRs.

steps $(0.4 \mathrm{fs} /$ step$)$ with the Nosé-Hoover thermostat. ${ }^{21}$ Then NEMD is performed for another $8 \times 10^{6}$ steps. Based on the criteria recommended for studying nonlinear thermal transport processes, ${ }^{22}$ we use the Berendsen thermostat ${ }^{23}$ as heat baths and maintain the same number of atoms adjacent to the two ends of the GNR at different $T$ ( $300 \mathrm{~K}$ for the hot end and 300 $\mathrm{K}-\Delta T$ the cold). The net heat current due to the bias $\Delta T$ is computed as $J=\left(\partial E_{\text {hot }} / \partial t+\partial E_{\text {cold }} / \partial t\right) / 2$, where $E_{\text {hot }}$ and $E_{\text {cold }}$ are the total energy that has been added to or subtracted from the atoms in the hot and cold thermostats, respectively.

The TR ratio $(\eta)$ is defined as

$$
\eta=\frac{\kappa_{\text {forward }}}{\kappa_{\text {backward }}}-1=\frac{J_{\text {forward }}}{J_{\text {backward }}}-1
$$

where the subscripts indicate whether the rectifier is operated at forward (heat flows left-to-right or wider-to-narrower) or backward bias. Figure $1 \mathrm{~b}$ shows $J$ as a function of the imposed $|\Delta T|$ in a trapezoidal-GNR. Sufficiently high $\Delta T$ is needed to initiate TR, and the direction of decreasing width $(\eta>0)$ is favored, which is consistent with previous studies on triangular ones. ${ }^{15,16}$ The temperature profiles for the forward and backward cases are plotted in Figure 1c, which demonstrates higher temperature gradient and hence lower $\kappa$ at narrower region. As the length $(L)$ and width $(w)$ of the trapezoidalGNR change by the same scale $(L=2 \sqrt{3} w)$ when its size changes, we also study T-shaped GNRs (the lower structure in Figure 1a) to evaluate which dimension has a more profound effect on TR. As shown in Figure 1d, $\eta$ of T-shaped GNRs decreases quickly with increasing $w$, but much more slowly with $L$, indicating that a sufficiently small lateral dimension is crucial for TR. In such narrow GNRs, phonons propagating in or close to the length direction are the predominant heat carriers, and the upper and lower edges are the dominant phonon scattering centers.

The strong lateral size dependence of TR motivates us to formally check if TR can exist in asymmetric structures of bulk size. For an asymmetric geometry with arbitrary dimension and shape as shown in Figure 2, of which the surfaces $S_{1}$ and $S_{2}$ are

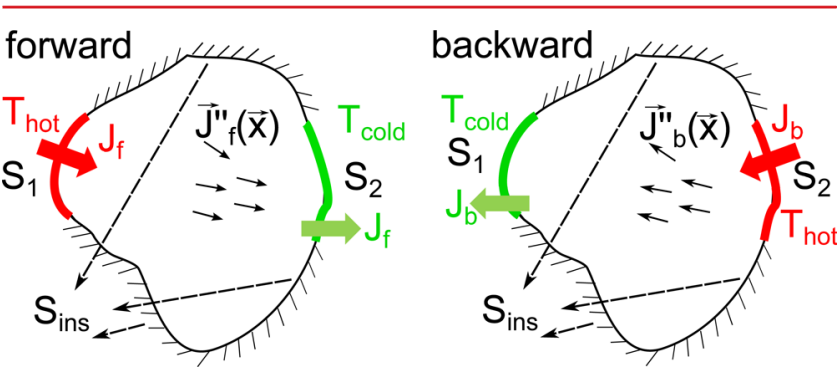

Figure 2. Schematic of the structure of an arbitrary shape applied with forward (left) and backward (right) temperature bias. $S_{1}$ and $S_{2}$ denote two surfaces maintained at $T_{\text {hot }}$ and $T_{\text {cold }}$ respectively for the forward direction and reversed for the backward direction. $S_{\text {ins }}$ denotes insulating (adiabatic) thermal boundaries. $J_{\mathrm{f}}$ and $J_{\mathrm{b}}$ are the total heat transfer rates, and $\vec{J}_{\mathrm{f}}{ }_{\mathrm{f}}(\vec{x})$ and $\vec{J}_{\mathrm{b}}{ }_{\mathrm{b}}(\vec{x})$ are the local heat fluxes.

maintained at $T_{\text {hot }}$ and $T_{\text {cold }}$, respectively, for the case of forward bias and vice versa for backward, and the remaining surfaces are insulated, we can analytically show that (the details are provided in the Supporting Information ${ }^{24}$ )

$$
\kappa\left(T_{\mathrm{f}}\right) \cdot \frac{\partial T_{\mathrm{f}}}{\partial \vec{x}}+\kappa\left(T_{\mathrm{b}}\right) \cdot \frac{\partial T_{\mathrm{b}}}{\partial \vec{x}}=0 \quad \text { or } \quad \vec{J}^{\prime \prime}{ }_{\mathrm{f}}(\vec{x})+\vec{J}^{\prime \prime}{ }_{\mathrm{b}}(\vec{x})=0
$$


which means that for any $N$-dimensional structure with anisotropic and temperature-dependent $\kappa$, at any position $x$, the local heat flux vectors $\left(\vec{J}_{f}^{\prime \prime}\right.$ and $\left.\vec{J}^{\prime \prime}{ }_{b}\right)$ in the forward and backward cases are of the same magnitude but in opposite directions. As an immediate result, the heat transfer rates for the two cases must be equal, that is, $J_{\mathrm{f}}=J_{\mathrm{b}}$. This is a strong conclusion of the absence of TR in bulk-size single-material homogeneous structures. To verify eq 2 , we have also solved the heat transfer problem for the systems in Figure 2 using the finite element method, and the results are provided as Supporting Information. ${ }^{24}$ It should be noted that for bulksize heterojunctions, $\kappa$ not only depends on temperature, but also on space, leading to the possibility of $\mathrm{TR}^{8,25}$ This is a different behavior from that of bulk-sized homogeneous structures of a single material.

The fact that single materials with a bulk lateral dimension cannot generate TR indicates that the phonon lateral confinement is necessary for TR to occur. This is a key finding of this work. In the phonon lateral confinement regime, the effects of lateral boundary on phonon properties are important. To explore why phonon lateral confinement is necessary, we examine several mechanisms that are known to be relevant in other TR systems.

We first examine the phonon spectra mechanism; ${ }^{16}$ that is, the amount of overlap $(\Psi)$ between the phonon spectra of the two sides of the structure changes after switching the thermostats. It has been commonly used to explain TR across interfaces between two dissimilar materials. ${ }^{4,11,26,27}$ The vibrational density of states (vDOS) in Figure $3 \mathrm{a}$ is computed (a)

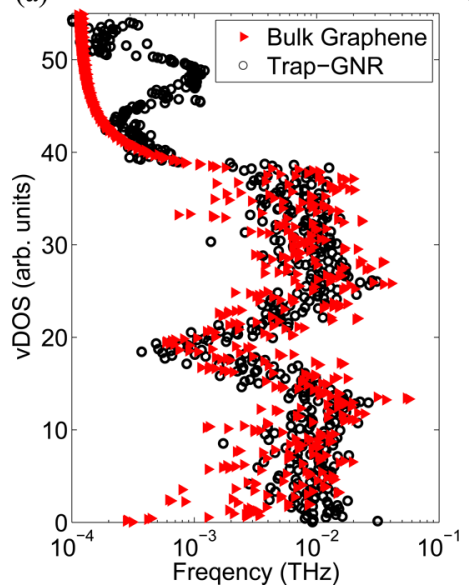

(b)

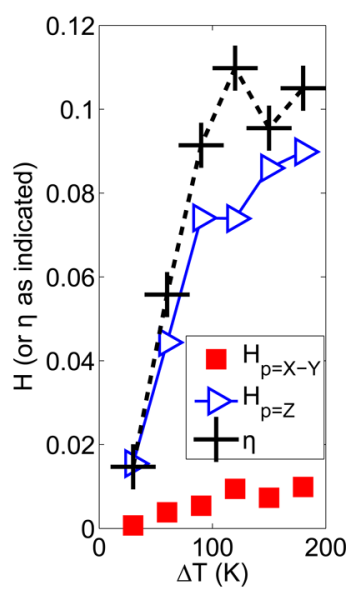

Figure 3. (a) Out-of-plane vibrational density of states. (b) $H$ and $\eta$ as a function of $\Delta T$.

as the Fourier transform of the out-of-plane component of the atomic velocity-velocity autocorrelation function. The vDOS is broadened when cutting a bulk graphene into a nanoribbon where edges are present. In parallel with eq 1 , we define $H=$ $\Psi_{\text {forward }} / \Psi_{\text {backward }}-1$ to quantify the change in phonon spectra overlap. Figure $3 \mathrm{~b}$ shows that the spectra overlaps are indeed different before and after switching the thermostats for both inplane $(p=X-Y)$ and out-of-plane $(p=Z)$ polarizations. We note that such difference is enabled by the small ribbon width, so that the local phonon spectra not only depends on temperature, but also on the width. The dependence of phonon spectra on the lateral size of nanostructures was observed previously. ${ }^{28}$ TR due to phonon spectra mismatch resulting from device-thermostat interactions was reported in a diamond nanopyramid. ${ }^{12}$ If the width increases to macroscopic size, the local phonon spectra will only depend on the temperature, and the phonon spectra overlap will be the same before and after switching the thermostats; thus TR vanishes. In contrast, the difference in phonon spectra overlap across interface in heterojunctions $s^{4,11,26,27}$ is enabled by two different materials, not by the confined lateral dimension. Hence, increasing the lateral dimension for an interface will not affect its TR.

The second possible mechanism is the inseparable dependence of $\kappa$ on $T$ and space, which has usually been used to explain TR in bulk materials, such as two-segment bars of different materials. ${ }^{5,7,25}$ Note that $\kappa$ is given as $\kappa \propto \int \mathrm{d} \omega v_{\mathrm{g}}(\omega)$ $\lambda(\omega) \hbar \omega D(\omega) \partial[n(\omega, T)] / \partial T$, where $\omega, v_{g}, \lambda, D$, and $n$ are the angular frequency, group velocity, mean free path, density of states, and the distribution function of phonons, respectively, and $\hbar$ is the reduced Planck constant. For our GNR, the phonon lateral confinement effect can make both $\lambda$ and $D$ dependent on the width, so that the local $\kappa$ becomes spacedependent. Such width-dependent $\kappa$ of GNR has been previously reported. ${ }^{29-32}$ Under this condition, heat conduction does not follow eq 2 , and TR can occur. Here we note that the inseparability in asymmetric GNRs is enabled by the phonon lateral confinement effect, ${ }^{31}$ not by using two different materials in the two-segment bars. As the width of the asymmetric GNR increases to bulk size, $\kappa$ only depends on $T$; thereby no TR can occur. Also, it is apparent that the inseparable thermal conductivity mechanism and the phonon spectra mechanism are not independent, since $\kappa$ depends partially on $D(\omega)$.

To explicitly show the role played by the small width on $\lambda(\omega)$, we explore the third possible mechanism-phonon localization, which has been suggested as a mechanism of TR in ref 14 for carbon nanocones and also reported in ref 12 for a diamond nanopyramid.

The participation ratio $\left(p_{\lambda}\right)$ characterizes phonons on a peratom basis and is defined for a specific mode $\lambda$ as $^{33}$

$$
p_{\lambda}^{-1}=N \sum_{i}\left(\sum_{\alpha} \varepsilon_{i \alpha, \lambda}^{*} \varepsilon_{i \alpha, \lambda}\right)^{2}
$$

which sums over all of the $N$ atoms numbered $i$, and polarizations of interest $(\alpha=X, Y$, or $Z) . \varepsilon_{i \alpha, \lambda}$ is the eigenvector component of the $\lambda$ th phonon mode. By definition, $p_{\lambda}$ varies between $O(1)$ for completely delocalized states and $O(1 / N)$ for completely localized ones. Herein we refer to $p<0.4$ as phonon localization, which ensures that all phonons in the bulk graphene are delocalized under this criteria. We can also picture the spatial distribution of a specific range $(\Lambda)$ of normal modes by $^{34}$

$$
\phi_{i \alpha, \Lambda}=\frac{\sum_{\lambda \in \Lambda} \varepsilon_{i \alpha, \lambda}^{*} \varepsilon_{i \alpha, \lambda}}{\sum_{1 \leq j \leq N} \sum_{\lambda \in \Lambda} \varepsilon_{j \alpha, \lambda}^{*} \varepsilon_{j \alpha, \lambda}}
$$

A larger value of $\phi_{i \alpha, \Lambda}$ indicates stronger localization of modes $\Lambda$ on the ith atom.

Figure $4 \mathrm{a}$ shows $p_{\lambda}$ for the bulk graphene and a T-shaped GNR. As we can see, $p_{\lambda}$ of the GNR is usually less than that of the bulk graphene, indicating stronger phonon localization in the former. The spatial distribution of the localized modes (LM) shown in Figures $4 b$ and $c$ reveals that LMs prefer edges. When heat flows in the direction of increasing width (Figure $4 c)$, more LMs can be found on the narrower end, which acts as 
(a)

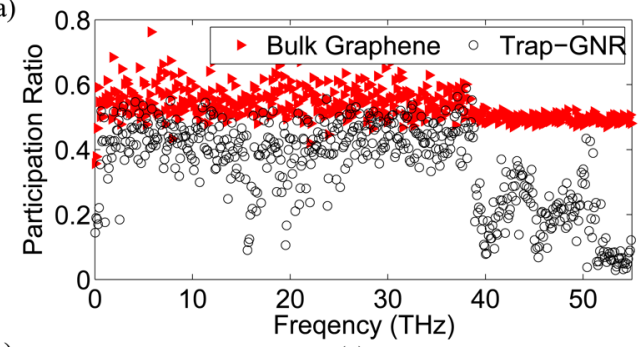

(b)

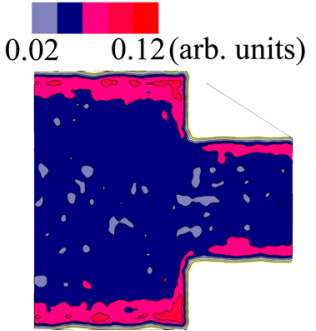

(c)

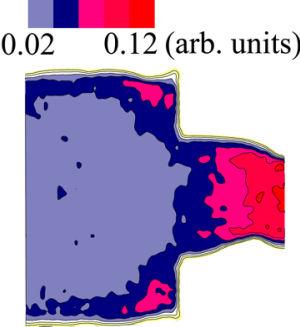

Figure 4. (a) Participation ratio of bulk graphene and T-shaped GNRs. Spatial distribution of localized modes when heat flows in the direction of (b) decreasing width (forward) and (c) increasing width (backward).

the bottleneck of thermal transport channel in such narrow GNRs. Therefore, delocalized modes have a narrower channel of propagation, and hence the effective $\kappa$ is reduced. Phonon localization can also be enhanced on the wider side when heat flows in the decreasing width direction (Figure 4b) but has less effect on thermal transport since the channel bottleneck is usually still the narrower end. Besides, LMs on edges can act as collision centers for other phonons, which alters $\lambda(\omega)$ and makes it space-dependent. In this sense, the phonon edge localization mechanism is correlated to the inseparable thermal conductivity mechanism through the space-dependent $\lambda(\omega)$. The above mechanism selects the direction of decreasing width as the favored direction of thermal rectifiers. When the GNR width increases to bulk size, three-phonon scattering dominates while edge scattering becomes unimportant; hence, TR disappears as depicted in Figure 1d.

To explore the origin of edge localization, we examine the spring constant spectra of the edge atoms and the interior ones in T-shaped GNRs. By calculating the restoring force $\Delta F$ after a small displacement $\Delta r$ of an atom, we can obtain the spring constant spectra of $\mathrm{C}$ atoms in different regions of a GNR with $\Gamma=-\Delta F / \Delta r$. The results are plotted in Figure 5. Compared with the interior atoms, the spring constant spectra of the edge atoms are broadened into higher and lower strength regions. The interior atoms have a much better translational symmetry than edge atoms that lose neighbors on one side. The lowfrequency tail results from the loss of neighbor atoms, ${ }^{28}$ which makes the environment softer; on the other hand, the highfrequency tail is caused by reconstructions of the edge $\mathrm{C}-\mathrm{C}$ bonds, ${ }^{35}$ which shortens the bonding length and makes the bonds harder. The distorted spring constant spectra place edge atoms in a quite different force environment from interior ones, which facilitates the generation of LMs. Moreover, since temperature affects the mean square displacement of $\mathrm{C}$ atoms directly, the spring constant disorder can be amplified by higher $T$. Consequently, higher $\Delta T$ and asymmetric geometry are needed to generate LMs asymmetrically and hence rectify heat flow, which can be confirmed by Figure $1 \mathrm{~b}$ and $\mathrm{d}$. As the nonequilibrium Green's function (NEGF) method uses (a)

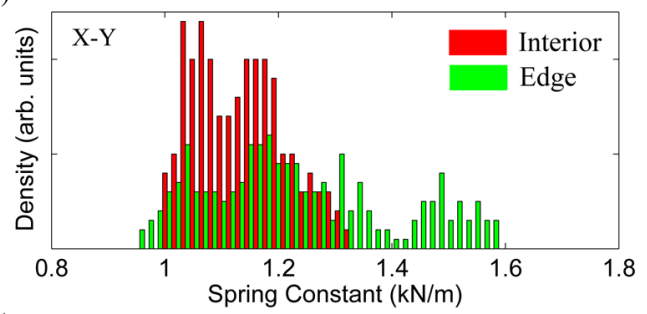

(b)

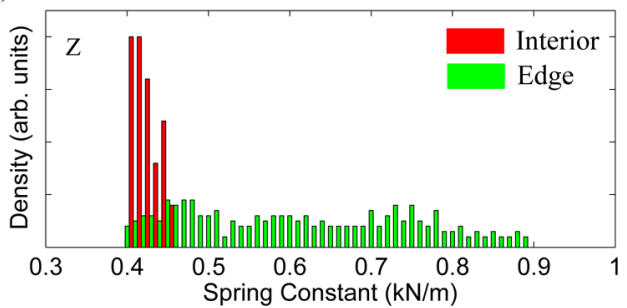

Figure 5. Spring constant spectra of (a) in-plane motion and (b) outof-plane motion of atoms.

constant spring constants due to the high computational cost requested by a $T$-dependent and anharmonic one, the previous NEGF study predicts no TR for two-terminal GNRs since no asymmetric phonon spectra like Figure $4 \mathrm{~b}$ and $\mathrm{c}$ can be generated, and multiple-terminal devices are needed which produces TR owing to a different mechanism. ${ }^{17}$

Phonon spectra of materials are usually broad, but the localization of phonons are selective to only certain modes. The broad spectra of phonons limits the efficiency of TR significantly below its electric counterpart which benefits from the fact that only a narrow spectra of electrons around the Fermi level contribute to conductance. In view of this analogy, TR can be potentially enhanced by narrowing the phonon spectra, such that all modes are localized in one direction of heat transport but delocalized in the opposite.

In the above, we have shown that TR in asymmetric GNRs can be explained by three possible mechanisms: phonon spectra overlap, inseparable dependence of $\kappa$ on $T$ and space, and phonon edge localization. All mechanisms require small GNR width so that phonons are confined. Therefore, phonon lateral confinement is the fundamental origin of $T R$ in such asymmetric homogeneous materials. Based on these findings, we have also checked other asymmetric nanostructures such as nonuniform nanowires, thin films, and quantum dots, as shown in Figure 6, which may also exhibit TR if they are in the phonon confinement regime, that is, the lateral dimension is small enough compared to $\lambda$. It should be noted that Figure $6 \mathrm{e}$ shows a structure similar to that proposed in ref 36 , but in our case the edges of both sides of the teeth are smooth, while in ref 36 the specularities of the two sides are different. In addition, our mechanism is significantly different from Lee et al.'s mechanism of phonon confinement. ${ }^{12}$ Our phonon edge confinement is in the device itself, while Lee et al.'s confinement focuses on the interaction between the device and contacts. These mechanisms can possibly be combined to achieve higher $\eta$. We note that a recent experimental study reported TR in large-size reduced graphene oxide, ${ }^{37}$ and the TR was attributed to the asymmetric geometry. Based on our findings, we argue that the asymmetric geometry is not sufficient and their TR is probably due to other mechanisms or experimental uncertainty. For example, graphene with both pristine and defected segments can sustain TR at a large size. ${ }^{11}$ 
(a) $\eta=0.39$

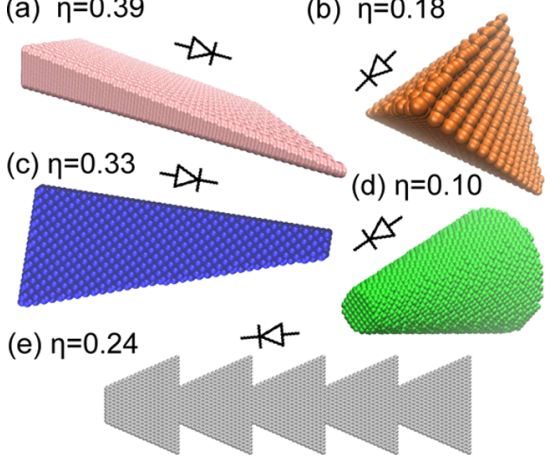

Figure 6. Homogeneous nanostructures as potential thermal rectifiers: (a) nano thin films with nonuniform thickness, (b) asymmetric quantum dots, (c) nano thin films with nonuniform width, (d) nonuniform nanowires, and (e) thermal rectifier series as proposed in literature, ${ }^{36}$ but with atomic smoothness for all edges. Directions of higher $\kappa$ and the value of $\eta$ are indicated. (a-d) have diamond lattice while (e) is cut from graphene, and all $\eta$ 's are calculated using the optimized Tersoff potential at $T=300 \mathrm{~K}$ and $\Delta T=90 \mathrm{~K}$.

To summarize, we have found that phonon confinement is the origin for TR in asymmetric homogeneous materials and that TR diminishes in bulk-sized ones. We have shown three possible mechanisms for TR in asymmetric GNRs: phonon spectra overlap, inseparable dependence of $\kappa$ on temperature and space, and phonon edge localization. These mechanisms are related to each other in a complicated manner. The widthdependent phonon spectra $D(\omega)$ can contribute to the spacedependence of $\kappa$. Also, the phonon edge localization can make $\lambda(\omega)$ space-dependent, which also contributes to the spacedependence of $\kappa$. The third mechanism explicitly shows the role played by the edges in the phonon confinement regime. When the lateral size of the device approaches the bulk limit, such edge effect is smeared out by three-phonon scatterings, and TR disappears. We also show that other asymmetric nanostructures, such as asymmetric nanowires, thin films, and quantum dots, of a single material are potential thermal rectifiers.

\section{ASSOCIATED CONTENT}

\section{S Supporting Information}

Analytical proof and numerical verifications of the absence of thermal rectification in bulk-size asymmetric single materials. This material is available free of charge via the Internet at http://pubs.acs.org.

\section{AUTHOR INFORMATION}

\section{Corresponding Author}

*E-mail: ruan@purdue.edu.

\section{Notes}

The authors declare no competing financial interest.

\section{ACKNOWLEDGMENTS}

This work was partially supported by the Air Force Office of Scientific Research (AFOSR). Hu, Chen, and Ruan are also associated with the Cooling Technologies Research Center (CTRC) at Purdue, an NSF industry/university cooperative research center, and the partial support was appreciated.

\section{REFERENCES}

(1) (a) Li, B.; Wang, L.; Casati, G. Phys. Rev. Lett. 2004, 93, 184301.

(b) Wang, L.; Li, B. Phys. Rev. Lett. 2007, 99, 177208.
(2) Liang, B.; Guo, X. S.; Tu, J.; Zhang, D.; Cheng, J. C. Nat. Mater. 2010, 9, 989-992.

(3) Chang, C. W.; Okawa, D.; Majumdar, A.; Zettl, A. Science 2006, 314, 1121-1124.

(4) Li, B.; Lan, J.; Wang, L. Phys. Rev. Lett. 2005, 95, 104302.

(5) Peyrard, M. EPL (Europhys. Lett.) 2006, 76, 49.

(6) Yang, N.; Li, N.; Wang, L.; Li, B. Phys. Rev. B 2007, 76, 020301.

(7) Dames, C. J. Heat Transfer 2009, 131, 061301.

(8) (a) Kobayashi, W.; Teraoka, Y.; Terasaki, I. Appl. Phys. Lett. 2009, 95, 171905. (b) Sawaki, D.; Kobayashi, W.; Moritomo, Y.; Terasaki, I. Appl. Phys. Lett. 2011, 98, 081915.

(9) Pereira, E. Phys. Rev. E 2011, 83, 031106.

(10) (a) Li, N.; Ren, J.; Wang, L.; Zhang, G.; Hänggi, P.; Li, B. Rev. Mod. Phys. 2012, 84, 1045-1066. (b) Roberts, N.; Walker, D. Int. J. Therm. Sci. 2011, 50, 648-662.

(11) Wang, Y.; Chen, S.; Ruan, X. Appl. Phys. Lett. 2012, 100, 163101.

(12) Lee, J.; Varshney, V.; Roy, A. K.; Ferguson, J. B.; Farmer, B. L. Nano Lett. 2012, 12, 3491-3496.

(13) Jiang, J.; Wang, J.; Li, B. EPL (Europhys. Lett.) 2010, 89, 46005.

(14) Yang, N.; Zhang, G.; Li, B. Appl. Phys. Lett. 2008, 93, 243111243111.

(15) Hu, J.; Ruan, X.; Chen, Y. P. Nano Lett. 2009, 9, 2730-2735.

(16) Yang, N.; Zhang, G.; Li, B. Appl. Phys. Lett. 2009, 95, 033107.

(17) Ouyang, T.; Chen, Y.; Xie, Y.; Wei, X. L.; Yang, K.; Yang, P.; Zhong, J. Phys. Rev. B 2010, 82, 245403.

(18) Here, by "single material", we mean a single and homogeneous material; hence, for instance, a system composed of both pristine and defected segments is not qualified as a "single material".

(19) Plimpton, S. J. Comput. Phys. 1995, 117, 1-19.

(20) Lindsay, L.; Broido, D. A. Phys. Rev. B 2010, 81, 205441.

(21) (a) Nosé, S. J. Chem. Phys. 1984, 81, 511-519. (b) Hoover, W. G. Phys. Rev. A 1985, 31, 1695-1697.

(22) Ai, B.-q.; Hu, B. Phys. Rev. E 2011, 83, 011131.

(23) Berendsen, H. J. C.; Postma, J. P. M.; van Gunsteren, W. F.; DiNola, A.; Haak, J. R. J. Chem. Phys. 1984, 81, 3684-3690.

(24) Detailed proof of the absence of thermal rectification in macroscopic homogeneous structures and the numerical verifications can be found in the Supporting Information.

(25) Go, D. B.; Sen, M. J. Heat Transfer 2010, 132, 124502.

(26) Zhang, X.; Hu, M.; Tang, D. J. Appl. Phys. 2013, 113, 194307.

(27) Pei, Q.-X.; Zhang, Y.-W.; Sha, Z.-D.; Shenoy, V. B. Appl. Phys. Lett. 2012, 100, 101901.

(28) Bao, H.; Ruan, X. L.; Kaviany, M. Phys. Rev. B 2008, 78, 125417.

(29) Guo, Z.; Zhang, D.; Gong, X.-G. Appl. Phys. Lett. 2009, 95, 163103.

(30) Aksamija, Z.; Knezevic, I. Appl. Phys. Lett. 2011, 98, 141919.

(31) Wang, Y.; Qiu, B.; Ruan, X. Appl. Phys. Lett. 2012, 101, 013101.

(32) Bae, M.-H.; Li, Z.; Aksamija, Z.; Martin, P. N.; Xiong, F.; Ong,

Z.-Y.; Knezevic, I.; Pop, E. Nat. Commun. 2013, 4, 1734.

(33) Bodapati, A.; Schelling, P. K.; Phillpot, S. R.; Keblinski, P. Phys. Rev. B 2006, 74, 245207.

(34) Chen, J.; Zhang, G.; Li, B. Nano Lett. 2010, 10, 3978-3983.

(35) Jiang, J.-W.; Wang, B.-S.; Wang, J.-S. Appl. Phys. Lett. 2011, 98, 113114.

(36) Roberts, N. A.; Walker, D. G. ASME Conf. Proc. 2011, 2011, T30053-T30053-9.

(37) Tian, H.; Xie, D.; Yang, Y.; Ren, T.-L.; Zhang, G.; Wang, Y.-F.; Zhou, C.-J.; Peng, P.-G.; Wang, L.-G.; Liu, L.-T. Sci. Rep. 2012, 2, 523. 